\title{
To Study Coronary Artery Stenosis on CT in Comparison with Catheter Angiography
}

\author{
Aastha Pruthi ${ }^{1}$, Anand M Rahalkar ${ }^{2}$, Manali Rahalkar ${ }^{3}$ \\ ${ }^{1}$ Ex- resident, Department of Radiology, Sassoon General Hospitals and BJ Medical College, Pune, ${ }^{2}$ Associate Professor, \\ Department of Radiology, Sassoon General Hospitals and BJ Medical College, Pune 400001, ${ }^{3}$ Associate Professor, Department \\ of Radiology, Sassoon General Hospitals and BJ Medical College, Pune 400001, India
}

Corresponding author: Dr. Anand M Rahalkar, 721/2B, Vikasnagar, Navi Peth, Pune 411030, India

DOI: http://dx.doi.org/10.21276/ijcmsr.2019.4.3.27

How to cite this article: Aastha Pruthi, Anand M Rahalkar, Manali Rahalkar. To study coronary artery stenosis on CT in comparison with catheter angiography. International Journal of Contemporary Medicine Surgery and Radiology. 2019;4(3):C118-C123.

\section{A B S T R A C T}

Introduction: Coronary artery disease is a significant cause of morbidity and mortality in present day scenario The aim of this study is to assess coronary artery stenosis on computed tomography and compare the results with standard conventional catheter angiography. The objectives of the study was to detect specificity and sensitivity of computed tomography angiogram to detect stenosis.

Material and methods: This was a prospective study including 50 patients from Sassoon general hospital who underwent computed tomography coronary angiogram as well as catheter angiography. After following ethical guidelines and taking informed consent, both the procedures were performed on separate days. Post processed images of computed tomography coronary angiograms were compared with conventional angiograms for degree of stenosis in each coronary artery. Additional information of artery dominance, type of plaques, bridging and anomalies was obtained.

Results: In this study, one of the methods-conventional angiography- is the gold standard to detect coronary stenosis. So sensitivity, specificity positive and negative predictive values of computed tomography angiograms to detect coronary artery stenosis were calculated. Sensitivity, specificity, positive and negative predictive value of computed tomography were 94.5\%, 95.2\%, 87.5\% and 95.5\%.

Conclusion: Computed tomography angiograms can be used as a screening test especially in patients with low to intermediate risk of coronary heart disease.

Keywords: Coronary, Stenosis, Computed Tomography, Catheter Angiography

\section{INTRODUCTION}

Coronary artery disease (CAD) has high prevalence. In the urban population, it is $7.9 \%$ to $11 \%$ above 20 years and $14.3 \%$ above 40 years of age. ${ }^{1,2,3} \mathrm{CAD}$ is the cause of death in $25.1 \%$ in urban population. ${ }^{4}$ Diagnosing and treating cardiovascular ailments has become important.

Conventional invasive coronary angiography currently remains the standard for the evaluation of CAD. ${ }^{5}$ The overall complication rate of conventional invasive coronary angiography is around $1.8 \%$ with mortality rate $0.1 \%$ but may be up to $0.55 \%$ in high-risk populations. ${ }^{6,7}$ Different risk stratification scores are in use like Framingham Risk Score and European Systematic Coronary Risk Evaluation. ${ }^{8,9}$ Such scores provide some prediction of who will have CAD and who will not. However, they have been found to perform poorly.

Role of computed tomography (CT) of coronary arteries was previously very limited due to longer scan times, slow gantry rotations and continuous pulsations. Recently Multiple detector/ Multislice CT have decreased scan times to few seconds using iso-volumetric image acquisitions and faster computing speeds. 128 slice CT can be used for diagnosis in wide range of heart rates. CT coronary angiography (CTCA) can also be applied for assessment of coronary artery anatomy, its variants/anomalies and other non-coronary artery cardiac indications.

Study aimed to access the role of coronary artery stenosis on CT coronary angiography in comparison with catheter angiography (CA) with the objectives to assess anatomy, dominance, presence and characterisation of plaques in coronary artery on computed tomography coronary angiography and to assess coronary artery stenosis in each of the artery as visualised on CT coronary angiography on comparison with catheter angiography and to detect specificity and sensitivity of CTCA.

\section{MATERIAL AND METHODS}

This was a prospective study done from June 2012 to June 2014 in Sassoon general hospital. Sample size is 50 patients.

Inclusion criteria: Patients coming for routine screening and getting CT coronary angiography (CTCA) and conventional angiography (CA) both. 
Exclusion criteria: patient not undergoing both the scans. Patient undergoing only calcium scores. Any symptomatic patients.

Scanning Protocol: After taking approval from institute ethics committee and informed consent, they were evaluated for CTCA finding. Subjects were scanned on Siemens 128 slice scanner. Patients having irregular heart rhythm or high heart rate were given beta blockers. For CT calcium score, plain scan was acquired at $120 \mathrm{Kv}, 100 \mathrm{mAs}, 3 \mathrm{~mm}$ slice thickness, $64 \times 0.6 \mathrm{~mm}$ collimation, field of view (FOV) of 200. Scan data was acquired in cranio-caudal direction. Matrix size of $512 \times 512$. All subjects with calcium score of $>400$ were advised catheter angiography. Patients having poor breath hold after beta blockers were excluded from the study. For CTCA, $80-100 \mathrm{ml}$ of non-ionic contrast-Iohexol $(350 \mathrm{mg} / \mathrm{ml})$ was injected at rate of 4.5 to $5.5 \mathrm{ml} / \mathrm{sec}$. Autotrigger technique was used with trigger kept on descending aorta, with threshold at $100 \mathrm{HU}$.Images were acquired during whole cardiac cycle. Scanning parameters employed during scanning were $-120 \mathrm{Kv}, 100 \mathrm{mAs}$, $1 \mathrm{~mm}$ slice thickness, FOV of 200 and $64 \times 0.6$ collimation. Retrospective ECG-gated reconstructions were done at various $\mathrm{R}-\mathrm{R}$ time intervals. B46f Heart-view sharp kernel and $1 \mathrm{~mm}$ slice thickness was used for reconstruction. Images were viewed in a cardiac window with window level at $200 \mathrm{HU}$ and window width of $600 \mathrm{HU}$.

The acquired data sets were evaluated in axial sections and also using various post-processing methods like multi-planar reconstruction (MPR), curved MPR, maximum intensity projection (MIP), surface shaded display (SSD) and volume rendering technique (VRT). Coronary circulation was evaluated for dominance. Then, individual coronary artery was also assessed for presence of any variation or anomalies. The involvement of vessel by calcified or soft (non-calcified) atherosclerotic plaques were categorized into no vessel diseased, single vessel diseased and multiple (i.e. more than one vessel) vessel diseased. The significant stenosis was defined as $>50-70 \%$ of luminal narrowing of coronary artery lumen. Catheter correlation was obtained in all subjects for comparison.

Catheter angiography (CA): Severe anaemia, uncontrolled hypertension, ventricular fibrillation, renal failure and congestive cardiac failure were contraindications. Serum creatinine was done in all patients. Contrast used was Iohexol-nonionic, water soluble and low osmolar-upto $50 \mathrm{ml}$.

Approach - Via Femoral artery with diagnostic catheter (jetking).

Angiograms:. Right anterior oblique caudal, AP, Cranial, LAO cranial, LAO caudal are the views for right side. LAO caudal and AP are views taken for the left side. One AP view for renals is also taken.

Additional evaluation of intracoronary pressure, pre and post stenosis is assessed. Distal flow /collateralization can also be visualized. If stent placement is to be done in the same setting, exact length and gauge of stent can be assessed. Post-stent placement revascularization can be documented.

\section{RESULTS}

Only 3 patients were under 45 years, rest all were above 45 years. $80 \%$ were males. Right dominant circulation was observed in 40 subjects (80\%). Left dominant circulation was seen in 7 subjects (14\%) and co-dominant circulation in 3 subjects $(6 \%)$.

Calcium Score: Out of 50 subjects, 24 subjects (48\%) had calcium score less than 80.14 of these (58\% of these) had non-significant stenosis and 10 (41\% of these) had significant stenosis. 26 (52\%) subjects had calcium score more than 80 . 9 of these (34.6\% of these) had non-significant stenosis and $17(65.4 \%$ of these) had significant stenosis. Three subjects having multiple vessels with significant stenosis had calcium score more than 80 , and one case had calcium score less than 80 as shown in table 1 .

Plaque: Highest number of plaques were found in LAD followed by RCA and circumflex. Type of plaque are reported as calcified, non-calcified/soft or mixed plaques Calcified being highest in prevalence, followed by mixed and soft plaque in each coronary artery. Figure $1 \mathrm{a}$ and $1 \mathrm{~b}$ shows mixed plaque in LAD. Figure $2 \mathrm{a}$ and $2 \mathrm{~b}$ show mid RCA

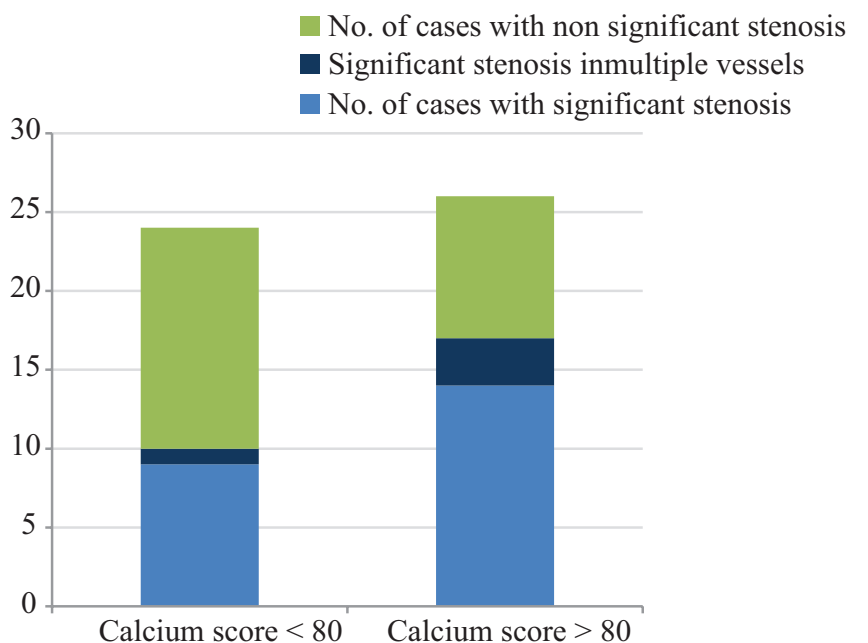

Table-1: This histogram shows more prevalence of significant stenosis among higher calcium

\begin{tabular}{|l|c|c|}
\hline $\begin{array}{l}\text { Table a) } \\
\text { CTCA }\end{array}$ & $\begin{array}{c}\text { CA } \\
\text { Positive }\end{array}$ & Negative \\
\hline RCA & & 0 \\
\hline Positive & 9 & 40 \\
\hline Negative & CA & \\
\hline $\begin{array}{l}\text { Table b) } \\
\text { CTCA }\end{array}$ & $\begin{array}{c}\text { Positive } \\
\text { LAD } \\
\text { Positive }\end{array}$ & $\begin{array}{c}\text { Negative } \\
2\end{array}$ \\
\hline Negative & 0 & 29 \\
\hline $\begin{array}{l}\text { Table c) } \\
\text { CTCA } \\
\text { CX }\end{array}$ & CA & \\
\hline Positive & 6 & 0 \\
\hline Negative & 19 \\
\hline \multicolumn{2}{|c|}{ Table-2: Depicts in toto results of the study. } \\
\hline
\end{tabular}




\begin{tabular}{|c|c|c|}
\hline & CATHETER ANGIO & CT ANGIO \\
\hline Contrast inj & Intraacoronary & Intravenous/periphery \\
\hline Contrast enhancement & Selective coronary & Complete vascular \\
\hline Image acquisition & Projection & Cross section(3D) \\
\hline Acquisition rate & 20 projections per heart cycle & 1 data set in 20 heart cycles \\
\hline Radiation exposure & 3-10mSv & 5-13 mSv \\
\hline Examination time & $>1 \mathrm{Hr}$ & $1-2 \mathrm{hr}$ \\
\hline In hospital time & $<1$ day & $1-2 \mathrm{hr}$ \\
\hline Spatial resolution & $0.1 \times 0.1$ xinfinite $\mathrm{mm}$ & $0.7 \times 0.7 \times 0.7 \mathrm{~mm}$ \\
\hline Temporal resolution & $<50 \mathrm{~ms}$ & $50-200 \mathrm{~ms}$ \\
\hline Advantages & $\begin{array}{l}\text { 1.Lesion quantification more accurate } \\
\text { 2.coronary flow, additional diagnostics (IVUS, } \\
\text { Pressure measurements) } \\
\text { 3. Intervention in the same setting possible }\end{array}$ & $\begin{array}{l}\text { 1.Minimally invasive } \\
\text { 2.Cardiac anatomy evaluated } \\
\text { 3.Plaque imaging } \\
\text { 4.Calcium score can be calculated }\end{array}$ \\
\hline Disadvantages & $\begin{array}{l}\text { Complications and discomfort related to arterial } \\
\text { puncture and coronary intubation }\end{array}$ & $\begin{array}{l}\text { Sensitive to arrhythmia, calcified vessels, poor } \\
\text { breath hold }\end{array}$ \\
\hline
\end{tabular}

\begin{tabular}{|l|c|c|c|}
\hline Authors & Right dominance & Left dominance & Co dominance \\
\hline Bezbaruah NK(2003) & $76 \%$ & $20 \%$ & $4 \%$ \\
\hline Kalpana R (2003) & $89 \%$ & $11 \%$ & - \\
\hline Present study & $84 \%$ & $14 \%$ & $2 \%$ \\
\hline \multicolumn{2}{|r|}{ Table-4: Distribution of dominance in different study. } \\
\hline
\end{tabular}

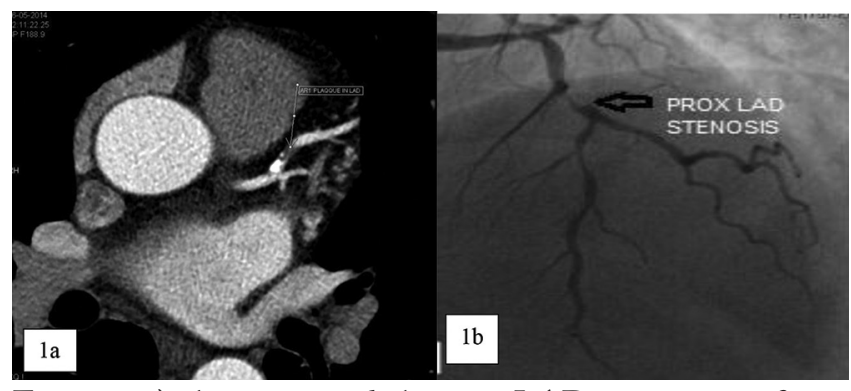

Figure-1a): shows a mixed plaque in LAD causing significant stenosis. 1b) confirms the findings on CA

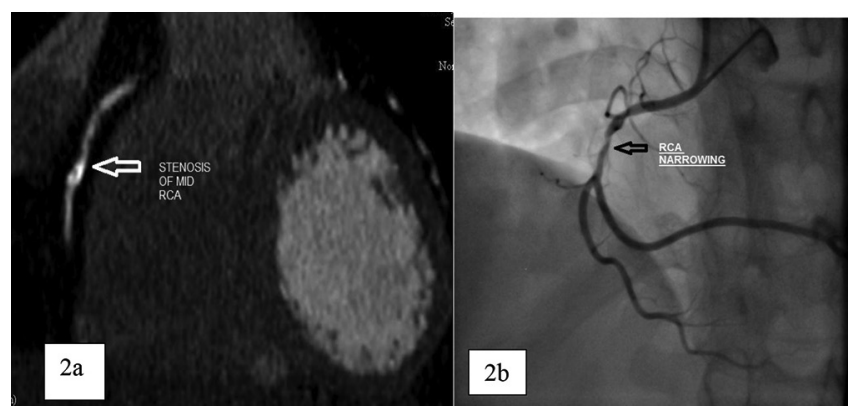

Figure-2a and $2 \mathrm{~b}$ : shows stenosis in mid RCA on CTCA and CA.

stenosis. Figure $3 \mathrm{a}$ and $3 \mathrm{~b}$ show focal calcified plaque.

Stenosis: Each coronary artery proximal, mid and distal segments were evaluated. Significant stenosis by definition was taken as $50-70 \%$ narrowing. Rest all cases were taken as non-significant narrowing. For LAD Sensitivity was100\%, Specificity 96.6, positive predictive value $95 \%$ and negative predictive value $100 \%$. For RCA_Sensitivity $88.8 \%$, specificity $95.1 \%$ positive predictive value $80 \%$, negative predictive value
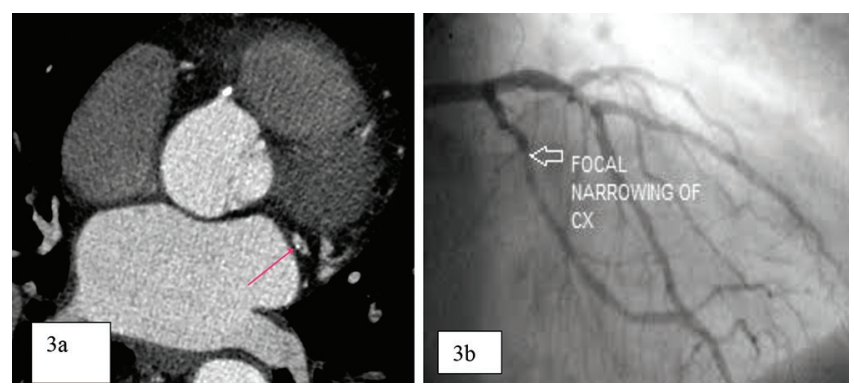

Figure-3a and 3b: Non significant focal narrowing in circumflex artery seen on CTCA and CA.

$97.5 \%$. For circumflex artery sensitivity $=87.5 \%$, specificity $95.2 \%$ positive predictive value $79 \%$ and negative predictive value $97.5 \%$. This is shown in Table 2.

Intoto sensitivity: $94.5 \%$ Intoto specificity $95.5 \%$ Intoto positive predictive value $87.5 \%$ Intoto negative predictive value $95.5 \%$ (Table 2 ).

Subjects with significant stenotic lesions in LAD, RCA and circumflex were 19,10 and 7 respectively and those with nonsignificant stenotic lesions were 31, 40 and 43 respectively. Left main coronary artery was normal in all cases except 1 .

Variants: Bridging of the coronary arteries was better appreciated on CTCA, with 10 subjects showing bridging. 9 of these where bridging of LAD. One of it was focal bridging of right coronary artery. Variation in origins of coronary arteries was seen in 1 case with right coronary artery taking origin from left coronary sinus. A case of ectasia of LAD artery with luminal expansion by more than 1.5 times.Type III LAD (beyond apex into posterior interventricular groove) was seen in 10 subjects out of 50 . 


\section{DISCUSSION}

CAD causes the largest healthcare burden to society. To decrease the morbidity and mortality of patients having coronary artery disease, it is necessary to diagnose and manage CAD at an earlier stage. The Framingham Heart Study is the origin of the term 'risk factor'. These can be modifiable and non-modifiable factors. Modifiable factors can be lifestyle and environmental factors Non modifiable factors are age, sex, hereditary factors i.e family history of coronary artery disease.

Age: Framinghams study suggest increased risk over age 45 years in men and over age 55 years in women. In our study too, these findings hold true. Almost all of the subjects having significant $\mathrm{CAD}$ belong to an age group more than 45 years. Those below the age of 45 years have predominantly non-significant coronary artery stenosis.

Gender: Overall men are more prone to atherosclerosis than women ${ }^{27}$ probably due to protective role of estrogen. Postmenopausal women have nearly the same risk as males of similar age group. In our study too there were $14 \%$ females and $86 \%$ males. Among these too, more percentage of males had significant coronary stenosis as compared to females.

With advancements in CT technology we now use CTCA for evaluation of coronary arteries. This can be done as an out-patient procedure without any special preparation, except for those routinely required prior to any other CT study with contrast. The ease and minimum invasive nature makes it an attractive tool for clinical application. Lesser complication rates as compared to conventional catheter angiography and simultaneous assessment of coronary artery calcium score (Modified Agatston score) also favor use of CT angiography of coronary arteries.

MSCT coronary angiograms are different from conventional angiograms as shown in the table. Conventional angiograms are projections of a vessel that has been enhanced with high concentration of contrast material. During injection of contrast, multiple images of this vessel are acquired throughout a number of cycles. In MSCT angiography, the cross sectional images of the coronary arteries are reconstructed during single phase of a number of consecutive heart cycles.

Dominance: The term right or left "Coronary Dominance" was used to show which coronary artery supplies the heart's diaphragmatic surface, based on the origin of the posterior interventricular artery (PIVA). Origin of the PIVA from the RCA was termed 'right dominance'; from the circumflex artery was called 'left dominance'. Origin from both the RCA and the circumflex artery was known as co dominant pattern.

Almost all authors have reported higher percentages of right dominance. Our study findings coincide with Indian as well as international study results in prevalence of dominance. It is important to know clinically about the dominance of artery, e.g. stenosis of circumflex needs to be treated more aggressively in a left dominant heart and vice versa is true for right dominant circulation.

Calcium Score: The prognostic value of coronary calcium has been studied in many studies. These studies indicate that calcium scoring can be used as risk factor, i.e a high calcium score is associated with relatively high risk of adverse coronary events. Absence of calcium is associated with low likelihood of advanced coronary disease and a very less likelihood of advanced coronary atherosclerosis. Greenland and Gazaino ${ }^{28}$ proved in their study that a calcium score less than 80 significantly reduced the risk of occurrence of coronary event in next 10 years i.e.have a lesser tendency to be associated with significant stenotic lesions. Our study also gives a larger portion of patients with significant stenosis belonging to calcium scores more than 80 . However scores less than 80 doesn't rule out presence of significant stenosis.

PLAQUE:LAD show highest prevalence of plaques, followed by RCA and circumflex. Left main artery shows lowest incidence of atherosclerosis in our study. This corroborates with the findings of above mentioned study, which also labels LAD, especially proximal and mid segments as most vulnerable to plaques. These can be calcified, non-calcified/ soft or mixed depending on morphology. The sensitivity of plaque detection depends on percentage of calcium in the plaque. Calcified plaques are found to be more stable and less prone to rupture. A study by Leber et $\mathrm{a}^{11}$ found that among asymptomatic patients, calcified plaques are highest in prevalence. Our study too, shows similar findings. Among all the plaques detected on CT coronary angiography, calcified plaques showed highest predominance in all the arteries (55-70\%).Whereas, soft plaques show more association in patients with angina pectoris and myocardial infarction as suggested by Mollet et al. ${ }^{15}$

Artery Stenosis: CT coronary angiography results showed a slight overestimation of significant stenosis on CTCA giving slightly lower positive predictive value. Optimal breath hold, regular heart rate artifacts due to motion and calcifications are many factors responsible for this. In our case, 2 false positive cases were due to excess calcium in the plaques. These are known facts described in Accuracy Trial by Budoff et al. ${ }^{22}$ As compared to Herzog C, Peter L. Zwerner, ${ }^{14}$ et al who did a study titled "Significant Coronary Artery Stenosis: Comparison on Per-Patient and Per-Vessel or Per-Segment Basis at 64-Section CT Angiography", found that on a persegment basis, upto $90 \%$ of all segments could be clearly evaluated. Stenoses of $50 \%$ or greater were detected with sensitivity, and specificity, respectively, of $89 \%$ and $91.5 \%$ on a per-vessel basis. Dewey et al also studied a total 30 number of patients, CT coronary and conventional angiography both were performed, which, together with quantitative analysis, served as the reference standard. Our findings match well with the conclusions of above mentioned studies. Most of the plaques detected were not causing significant stenosis. This was expected, as our study included only asymptomatic patients.

Variants: Highly variable observations are reported regarding myocardial bridging, ranging from 1.5 to $1.6 \%$ in catheter angiography study to 18 to $30 \%$ in CT coronary angiographic studies. It is well appreciated by on CT coronary angiography. Catheter angiography is less sensitive. Anomalous origin of $\mathrm{RCA}$ is a benign anomaly in which right coronary artery 
taking origin from left aortic sinus. It is appreciated well on both CT and catheter angiography.

Ectasia is dilatation of arterial lumen by 1.5 times or more than the original. It was appreciated both on CT and catheter angiography.

\section{CONCLUSION}

Catheter angiography remains the gold standard for diagnosis of significant stenosis, as diagnosis and treatment can be done in the same setting. In all, CTCA demonstrates good negative predictive value. Also sensitivity, specificity and positive predictive value are such that it can be used as a good screening test in subjects with low to intermediate likelihood of coronary artery disease. And those depicting normal coronaries can avoid the hassles of catheter angiography and the costs associated. Characterisation of plaque and detection of anomalies is an additional technically useful information for any intervention later on. In case of malignant anomalies, preventive measures can be advised.

\section{REFERENCES}

1. Gupta R, Gupta VP. Metanalysis of CHD prevalence in India, Indian heart J. 1996; 48(1): 41-45.

2. The Chennai urban population study (CUPS No. 5) Journal of the American College of Cardiology, Volume 38, Issue 3, Pages 682-687.

3. Ramchandra A, Jali MV, Mohan V, Snehalatha C, Viswanathan M. Clustering of cardiovascular risk factors in urban Asian Indians, Diabetes Care - 1998;21 (3): $967-971$.

4. ICMR (2004) - Assessment of burden of noncommunicable diseases - Final report.

5. Koen Nieman, Filipo Cademariti. Reliable noninvasive coronary angiography with fast sub-millimetre multislice spiral computed tomography, Circulation, 2002; 106 (5):2051-2054.

6. Harish A, Khatri P, Priyadarshini H, Selvakumar S, Arunkumar N, Sivakumar A, et al. Accuracy of 64-slice Coronary CT angiography in predicting percentage luminal stenosis. Indian Heart J 2008; 60 (2): 296-301.

7. Hemant Telkar. Coronary calcium score: Indian scenario \& experience. Indian J Radiology \& Imaging, vol.17, issue1, pg no.43-48.

8. Ferencik M, Ropers D, Abbara S, Cury RC, Hoffmann U, Nieman Ket al. Diagnostic Accuracy of Image Post processing Methods for the Detection of Coronary Artery Stenosis by Using Multidetector CT, RADIOLOGY 2007;243 (4): 696-702.

9. Rahalkar Anand, Rahalkar Mukund. Pictorial Essay: coronary artery variants \& anomalies. Indian J Radiology \& Imaging, vol.19, issue1, pg no.49-53.

10. Hansel J. Otero, Michael L. Steigner, Rybicki FJ. The "Post- 64" Era of Coronary CT Angiography: Understanding New Technology from Physical Principles, Radiol Clin N Am 47 2009;47 (3): 79-90.

11. Leber AW, Knez A, von Ziegler F, Becker A, Nikolaou K, Paul S et al. Quantification of obstructive and nonobstructive coronary lesions by 64 -slice computed tomography: a comparative study with quantitative coronary angiography and intravascular ultrasound. J Am Coll Cardiol 2005; 46 (6):147-54.
12. Zimmermann E, Deissenrieder F, Laule M, Dübel HP, Schlattmann P, Knebel F, et al. Noninvasive coronary angiography by 320-row computed tomography with lower radiation exposure and maintained diagnostic accuracy: comparison of results with cardiac catheterization in a head-to-head pilot investigation. Circulation. 2009;120(10):867-75.

13. Sun K, Han RJ, Cui LF, Zhao RP, Ma LJ,Wang LJ, et al. Feasibility and Diagnostic Accuracy for Assessment of Coronary Artery Stenosis of Prospectively ECGgated High-pitch Spiral Acquisition Mode Dualsource CT Coronary Angiography in Patients with Relatively Higher Heart Rates: in Comparison with Catheter Coronary Angiography. Chin Med Sci J 2013;27(4):213-9

14. Herzog C, Zwerner PL, Doll JR, Nielsen CD, Nguyen SA, Savino G, et al Significant Coronary Artery Stenosis: Comparison on Per-Patient and Per-Vessel or Per-Segment Basis at 64-Section CT Angiography. Radiology. 2007;244(1):112-20.

15. Pugliese F, Mollet NR, Runza G, van Mieghem C, Meijboom WB, Malagutti P, et al. Diagnostic accuracy of non-invasive 64-slice CT coronary angiography in patients with stable angina pectoris, Eur Radiol 2006; 16 (1): 575-582.

16. Prakash A, Ahlawat K, Kaul UA, Tyagi S, Aggarwal B, Rajan S et al. Accuracy of 64-slice CT Coronary Angiography: Our Initial Experience. Ind Heart J 2008; 60: 287-295.

17. Jones CM, Athanasiou T, Dunne N, Kirby J, Aziz O, Haq A, et al. Multi-Detector Computed Tomography in Coronary Artery Bypass Graft Assessment: A MetaAnalysis. Ann Thorac Surg 2007; 83 (5):341- 8.

18. Herzog BA, Husmann L, Burkhard N, Gaemperli O, Valenta I, Tatsugami F,et al. Accuracy of low-dose computed tomography coronary angiography using prospective electrocardiogram-triggering: first clinical experience. European Heart Journal 2008;29:30373042.

19. Anders K, Achenbach S, Petit I, Daniel WG, Uder M, Pflederer T. Accuracy of automated software-guided detection of significant coronary artery stenosis by CT angiography: comparison with invasive catheterisation. Eur Radiol 2013;23(5):1218-25.

20. Becker CR, Knez A, Leber A, Treede H, Ohnesorge B, Schoepf UJ et al. Detection of coronary artery stenosis with multislice helical CT angiography.J comput assist tomogr 2002;26(5):750-5.

21. Hoffmann MH, Shi H, Schmitz BL, Schmid FT, Lieberknecht M, Schulze R, et al. Non-invasive coronary angiography with multislice computed tomography. JAMA 2005; 293 (1):2471- 8.

22. Budof MJ, Dowe D, Min J; VCT Study Group. Results From the Prospective Multicenter ACCURACY (Assessment by Coronary Computed Tomographic Angiography of Individuals Undergoing Invasive Coronary Angiography) Trial. J Am Coll Cardiol 2008; 52:1724-32.

23. Miller JM, Rochitte CE, Dewey M, Arbab-Zadeh A, Niinuma H, Gottlieb I, et al. Diagnostic Performance of Coronary Angiography by 64-Row CT. N Engl J Med 
2008; 359 (6):2324-36.

24. Yamanaka $\mathrm{O}$, Hobbs RE. Coronary artery anomalies in 595 patients undergoing coronary arteriography. Cathet Cardiovasc Diagn 1990; 21 (3):28 - 40.

25. Topas O, DeMarchena EJ, Perin E, Sommer LS, Mallon SM, Chahine RA. Anomalous coronary arteries: angiographic findings in 80 patients. Int J Cardiol 1992; 34(3):129-38

26. Coronary artery anomalies: a comprehensive approach. Philadelphia: Lippincott Williams \& Wilkins; 1999. p. 27-150.

27. Erol C1, Seker M. The prevalence of coronary artery variations on coronary computed tomography angiography. Acta Radiol. 2012;53(3):278-84.

28. Greenland P, Gaziano JM. Selecting asymptomatic patients for coronary computed tomography or electrocardiographic exercise testing. N Engl J Med. 2003;349(5):465-73.

Source of Support: Nil; Conflict of Interest: None

Submitted: 13-07-2019; Accepted: 07-08-2019; Published online: 04-09-2019 